\title{
Benefits of physical therapy for people living with hemophilia
}

\author{
Kazuko Kikuchi ${ }^{1,2}$, Toshiharu Komachi ${ }^{2}$, Yoshinori Honma ${ }^{2}$, Junko Fujitani ${ }^{2, *}$ \\ ${ }^{1}$ Japan Foundation for AIDS Prevention, Tokyo, Japan; \\ ${ }^{2}$ Department of Rehabilitation Medicine, National Center for Global Health and Medicine, Tokyo, Japan.
}

\begin{abstract}
This crossover study investigated effects of physical therapy (PT) on motor function in patients with hemophilia infected with human immunodeficiency virus (HIV) due to treatment with non-heat-treated blood products. Patients were randomly divided into a PT-first group (PT once monthly for 6 months, then only home exercise (HE) for 6 months) and an HE-first group (HE for 6 months, then PT once monthly for 6 months). Carryover, period, and treatment effects were examined. Carryover effect was observed for flexion muscle strength of the right hip joint and fast walking cadence. Period effect was observed for extension Range of motion (ROM) of the left hip joint, dorsiflexion of the right ankle joint, and fast walking cadence. PT tended to be effective for abduction of the left shoulder joint and fast walking cadence. Compared with HE, PT tended to contribute to improving ROM of the shoulder joints and fast walking.
\end{abstract}

Keywords: home exercise, crossover, cadence, walking

Physical therapy programs for persons with hemophilia were introduced in the Southern California area in 1959 (1). By the 1960s, the importance of exercise therapy was demonstrated, with the effects of preventing joint deformity and bleeding, and relieving pain $(1,2)$. However, patients with hemophilia do not have much experience with exercising, and many do not perform daily active physical exercise (3).

In a study by Goto et al. $(3,4)$, the effects of home exercise were examined and demonstrated to improve physical function and activities of daily living (ADL), and to prevent bleeding. Also, in terms of the necessity and effects of physical therapy, Wittmeier (5) showed that physical therapy helped in prevention and recovery from bleeding and improved levels of physical activity. Forsyth et al. (6) also showed that in order for hemophilia patients to start exercising, physical therapists who can properly evaluate the exercise are indispensable. Therefore, in the present study, we investigated the effects of physical therapy in patients with hemophilia infected with human immunodeficiency virus (HIV) due to treatment with non-heat-treated blood products, by comparing the effects of home exercise with versus without oncemonthly physical therapy delivered by a physical therapist for 6 months.

This study was conducted at the Department of Rehabilitation Medicine, National Center for Global Health and Medicine between October 2015 and November 2017. Inclusion criteria were ability to undergo evaluation of joint range of motion, muscle strength, grip strength, and $10-\mathrm{m}$ walking, and provision of informed consent to participate in the study. Ability to undergo the evaluation of $10-\mathrm{m}$ walking was not an essential requirement. Exclusion criteria were hemarthrosis, intraarticular bleeding, intramuscular bleeding, and unhealed fractures on the day of evaluation.

Participants were divided into 2 groups by the envelope method, and an unblinded crossover study was conducted. One group was the physical therapyfirst group, and the other was the home exercise-first group. Both groups underwent initial evaluation. After that, the physical therapy-first group received physical therapy once monthly in the first 6 months (Period I), performed home exercise using an instruction leaflet without monthly interventions by a physical therapist in the following 6 months (Period II), and then underwent final evaluation. The home exercise-first group performed home exercise in Period I, received physical therapy once monthly in Period II, and then underwent final evaluation. Home exercise and physical therapy were performed in the same way in the home exercisefirst group as in the physical therapy-first group.

Evaluation items were joint range of motion (flexion and abduction of the shoulder joint; flexion, extension, pronation, and supination of the elbow joint; flexion, extension, and abduction of the hip joint; straight leg raising (SLR); flexion and extension of the knee joint; flexion and dorsiflexion of the ankle), muscle strength 
(flexion, extension, and abduction of the hip joint; extension of the knee joint), and 10-m walking (normal and fast walking). Muscle strength was evaluated using a handheld dynamometer $\mu$ TasF-1 (Anima Corp., Tokyo, Japan) using the method developed by Hirasawa et al. (7). In 10-m walking, participants were instructed to walk at 2 speeds, normal and fast walking, and the speed, cadence, and stride length were analyzed.

Physical therapy included $i$ ) confirmation of the participant's status at 1 month (i.e., status of activity, bleeding and pain, and exercise); ii) confirmation and correction of stretch movements conducted at home; iii) range of motion training on several joints, depending on the evaluation result, by a physical therapist; $i v$ ) confirmation and correction of the form of muscle strength training conducted at home; v) guidance on changing the content of muscle strength training conducted at home (exercise menu and loads); and vi) confirmation and guidance on walking, and ascending and descending stairs.

Regarding home exercise, in accordance with the instruction leaflet, which was developed for patients with hemophilia (8), an exercise menu was prepared for each participant based on the results of motor function evaluation.

Differences in age, body mass index, range of motion, muscle strength, and walking ability at the start of the study between the physical therapy-first group and the home exercise-first group were examined using unpaired $t$-test. The crossover results were assessed using the $t$-test for period effect, treatment effect, and carryover effect on each evaluation items as described previously (9). Briefly, carryover effect was tested on the Period (I+II) data in both groups; treatment effect on Period (I-II)/2 data in both groups; and period effect on Period (I-II)/2 data in the physical therapyfirst group, while on Period (II-I)/2 data in the home exercise-first group. Analyses were performed using SPSS ver. 26 (IBM, Armonk, NY, USA).

This study was approved for central review by the Ethics Review Committee of the National Center for Global Health and Medicine (approval number, NCGM-G-003242-00), and appropriate ethics procedures were followed at each participating facility.

After excluding participants who dropped out of the study, the remaining participants were all men: 11 in the physical therapy-first group (mean age 51.9 years, mean height $171.83 \mathrm{~cm}$, mean weight $61.87 \mathrm{~kg}$ ) and 7 in the home exercise-first group (mean age 53.3 years, mean height $175 \mathrm{~cm}$, mean weight $59 \mathrm{~kg}$ ). There was no difference in age or BMI between the groups.

The following items were excluded from the analysis because the baseline measurements were significantly higher in the physical therapy-first group than in the home exercise-first group: flexion range of motion of the left shoulder joint, abduction range of motion of both hip joints, right SLR, and left SLR.
Table 1. The p-values for carryover effect, treatment effect, and period effect

\begin{tabular}{|c|c|c|c|}
\hline Range of motion & $\begin{array}{c}\text { Carryover } \\
\text { effect }\end{array}$ & $\begin{array}{c}\text { Treatment } \\
\text { effect }\end{array}$ & $\begin{array}{c}\text { Period } \\
\text { effect }\end{array}$ \\
\hline \multicolumn{4}{|l|}{ Right shoulder joint } \\
\hline Flexion & 0.195 & 0.147 & 0.421 \\
\hline Abduction & 0.773 & 0.444 & 0.172 \\
\hline \multicolumn{4}{|l|}{ Left shoulder joint } \\
\hline Abduction & 0.540 & 0.052 & 0.182 \\
\hline \multicolumn{4}{|l|}{ Right elbow joint } \\
\hline Flexion & 0.872 & 0.728 & 0.324 \\
\hline Extension & 0.142 & 0.983 & 0.356 \\
\hline Pronation & 0.848 & 0.638 & 0.532 \\
\hline Supination & 0.547 & 0.126 & 0.932 \\
\hline \multicolumn{4}{|l|}{ Left elbow joint } \\
\hline Flexion & 0.962 & 0.923 & 0.086 \\
\hline Extension & 0.245 & 0.605 & 0.155 \\
\hline Pronation & 0.468 & 0.488 & 0.488 \\
\hline Supination & 0.582 & 0.468 & 0.107 \\
\hline \multicolumn{4}{|l|}{ Right hip joint } \\
\hline Flexion & 0.116 & 0.511 & 0.511 \\
\hline Extension & 0.074 & 0.529 & 0.056 \\
\hline \multicolumn{4}{|l|}{ Left hip joint } \\
\hline Flexion & 0.78 & 0.188 & 0.067 \\
\hline Extension & 0.592 & 0.978 & $0.023 *$ \\
\hline \multicolumn{4}{|l|}{ Right knee joint } \\
\hline Flexion & 0.135 & 0.808 & 0.223 \\
\hline Extension & 0.204 & 0.668 & 0.267 \\
\hline \multicolumn{4}{|l|}{ Left knee joint } \\
\hline Flexion & 0.912 & 0.275 & 0.886 \\
\hline Extension & 0.586 & 0.505 & 0.685 \\
\hline \multicolumn{4}{|l|}{ Right ankle joint } \\
\hline Dorsiflexion & 0.825 & 0.33 & $0.003 *$ \\
\hline Plantar flexion & 0.804 & 0.144 & 0.601 \\
\hline \multicolumn{4}{|l|}{ Left ankle joint } \\
\hline Dorsiflexion & 0.167 & 0.814 & 0.31 \\
\hline Plantar flexion & 0.593 & 0.694 & 0.098 \\
\hline Muscle strength & $\begin{array}{c}\text { Carryover } \\
\text { effect }\end{array}$ & $\begin{array}{c}\text { Treatment } \\
\text { effect }\end{array}$ & $\begin{array}{c}\text { Period } \\
\text { effect }\end{array}$ \\
\hline \multicolumn{4}{|l|}{ Right hip joint } \\
\hline Flexion & $0.009 *$ & 0.691 & 0.368 \\
\hline Extension & 0.146 & 0.782 & 0.056 \\
\hline Abduction & 0.13 & 0.477 & 0.428 \\
\hline \multicolumn{4}{|l|}{ Left hip joint } \\
\hline Flexion & 0.144 & 0.666 & 0.565 \\
\hline Extension & 0.279 & 0.231 & 0.87 \\
\hline Abduction & 0.385 & 0.403 & 0.506 \\
\hline \multicolumn{4}{|l|}{ Right knee joint } \\
\hline Extension & 0.291 & 0.522 & 0.244 \\
\hline \multicolumn{4}{|l|}{ Left knee joint } \\
\hline Extension & 0.973 & 0.199 & 0.136 \\
\hline \multicolumn{4}{|l|}{ Normal walking } \\
\hline Walking speed & 0.106 & 0.811 & 0.138 \\
\hline Stride length & 0.434 & 0.469 & 0.769 \\
\hline Walking cadence & 0.327 & 0.447 & 0.816 \\
\hline \multicolumn{4}{|l|}{ Fast walking } \\
\hline Walking speed & 0.108 & 0.364 & 0.534 \\
\hline Stride length & 0.329 & 0.636 & 0.117 \\
\hline Walking cadence & $0.092 *$ & 0.091 & $0.009 *$ \\
\hline
\end{tabular}

*Asterisks denote statistical significance. A $p$-value of 0.1 was considered significant for carryover effect, while that of 0.05 for others.

Table 1 shows $p$-values for carryover effect, treatment effect, and period effect on range of motion, muscle strength and walking ability. Carryover effect was observed for flexion muscle strength of the right hip joint and cadence in fast walking. Period effect was observed for extension range of motion of the left hip joint, dorsiflexion range of motion of the right ankle joint, and cadence in fast walking. A significant 
treatment effect was not observed, but physical therapy tended to be effective for abduction range of motion of the left shoulder joint and cadence in fast walking.

In patients with hemophilia, hemarthrosis is accompanied by synovitis and arthropathy, resulting in muscle atrophy around the joint. Tiktinsky et al. (10) pointed out that excessive rest after bleeding causes muscle atrophy and joint contracture, resulting in a vicious cycle of rebleeding and impairment in ADL. To break this vicious cycle, physical therapy and exercise are recommended for patients with hemophilia $(1,2,11-14)$.

Tiktinsky et al. (10) demonstrated that continuous resistance training over 1 to 2 years resulted in increased muscle strength around the knees and elbows, prevention of bleeding, and pain relief in 2 adult patients with severe hemophilia. Hilberg et al. (15) reported that a 6-month-long physical training program in 9 adult patients with severe hemophilia resulted in a 34\% increase in lower limb muscle strength and an improvement in proprioception and coordination. Goto et al. (4) examined the effects of 8-week home exercise in 32 adult patients with hemophilia. Effects of home exercise were compared between 2 groups: one group simply performed exercises and the other performed exercises that incorporated self-monitoring. Results showed no difference in range of motion, balance ability, and walking speed, regardless of whether exercises incorporated self-monitoring. As described above, there are some reports on exercise therapy in patients with hemophilia; however, to our knowledge, no study to date has compared the effects of physical therapy with the effects of home exercise. In 2017, Ruben et al. (16) examined the effects of educational intervention together with home exercise conducted over 15 weeks in patients with hemophilia. However, the evaluation items were pain and quality of life, and motor function was not examined in detail. The outpatient physical therapy in the present study was not limited to manual training of range of motion or muscle strengthening, and each session also included evaluation of motor function and instruction on self-training based on the evaluation results for each patient.

A washout period was not included in this study, but a carryover effect was observed for only flexion muscle strength of the right hip joint and cadence in fast walking. On the other hand, a period effect was observed for extension range of motion of the left hip, dorsiflexion range of motion of the right ankle, and cadence in fast walking, suggesting that left hip extension and right ankle dorsiflexion worsens and cadence in fast walking improves over time. The treatment effect on fast walking was not significant, but showed a tendency toward improvement with physical therapy $(p=0.091)$.

Fast walking ability is a practical requirement in daily living (17). Goto et al. demonstrated improvement in walking, but whether the walking speed was normal or fast was not described (3). The present study examined both normal walking and fast walking, and found improvement in cadence in fast walking.

The exercise menu in this study was designed for each patient based on the results of motor function evaluation. Use of such personalized intervention in this study showed a tendency toward improvement of walking at practically important speed, which was not reported previously. This suggests that accurate evaluation and provision of instruction and a self-training exercise menu based on the evaluation results, rather than simply providing range of motion training or muscle strengthening training manually, may be more effective in physical therapy to improve motor function in patients with hemophilia and HIV infection.

One of the limitations in this study was that the way patients performed home exercise was not followed in detail, and their adherence to our instruction was judged based on their verbal reports only. Greene et al. reported that it is difficult to achieve favorable adherence to home exercise in patients with hemophilia due to their fear of bleeding (18). A method for accurately monitoring the performance of home exercise is needed in order to provide more effective instructions in the future. Another limitation is that a washout period was not included in this study. Consequently, a carryover effect on results in Period II was observed, albeit only for a few measurement items. To validate the effect of physical therapy in the future, comparisons should be performed in studies with an adequate washout period.

Funding: This study was supported by a Ministry of Health, Labour and Welfare Policy Research Grant and the AIDS Policy Research Project (H30-AIDSDesignated-002).

Conflict of Interest: The authors have no conflicts of interest to disclose.

\section{References}

1. Boone DC. A total program for the patient with hemophilia. II. Physical therapy aspects related to orthopaedic and neurologic residuals of bleeding. Phys Ther. 1966; 42:1272-1281.

2. Austin E, Rolland W, Clausen D. Use of physical therapy modalities in the treatment of orthopedic and neurologic residuals in hemophilia. Arch phys Med Rehabil. 1961; 42:393-397.

3. Goto M, Takedani H, Kubota M, Haga N, Nitta S. The effect of a home exercise program for patients with hemophilia. Off J Jpn Prim Care Assoc. 2014; 37:22-26. (in Japanese)

4. Goto M, Takedani H, Haga N, Kubota M, Ishiyama M, Ito S, Nitta O. Self-monitoring has potential for home exercise programmes in patients with haemophilia. Haemophilia. 2014; 20:e121-e127.

5. Wittmeier K, Mulder K. Enhancing lifestyle for individuals with haemophilia through physical activity 
and exercise: the role of physiotherapy. Haemophilia. 2007; 13(Suppl 2):31-37.

6. Forsyth AL, Quon DV, Konkle BA. Role of exercise and physical activity on haemophilic arthropathy, fall prevention and osteoporosis. Hemophilia. 2011; 17:e870876.

7. Hirasawa Y, Hasegawa T, Sasa M, Yamasaki H. The validity of the isometric knee extension muscle strength by hand held dynamometer. Gen Rehabil. 2005; 33:375377. (in Japanese)

8. Kakinuma A, Mizukoshi E. Exercises for people with hemophilia. https://www.habatakifukushi.jp/pdf/exercise. $p d f$ (accessed May 17, 2021). (in Japanese)

9. Origasa H. Crossover study planning and analysis. Jpn Pharmacol Ther. 2016; 44:1261-1269. (in Japanese)

10. Tiktinsky R, Falk B, Heim M, Martinovitz U. The effect of resistance training on the frequency of bleeding in haemophilia patients: a pilot study. Haemophilia. 2002; 8:22-27.

11. Beardsley DS. Hemophilia. In: Sports and Exercise for Children with Chronic Health Conditions (Goldberg B, ed.). Human Kinetics, Champaign, IL, 1995; pp. 301-310.

12. Buzzard BM, Jones PM. Physiotherapy management of haemophilia. Physiotherapy. 1988; 74:221-226.

13. Buzzard BM. Sports and hemophilia: antagonist or protagonist. Clin Orthop Relat Res. 1996; 328:25-30.

14. McLarin LG, Heldrich FT. Hemophilia and sports: guidelines for participation. Physician Sportsmed. 1990; 18:73-80.
15. Hilberg T, Herbsleb M, Puta C, Gabriel HH, Schramm W. Physical training increases isometric muscular strength and proprioceptive performance in haemophilic subjects. Haemophilia. 2003; 9:86-93.

16. Cuesta-Barriuso R, Torres-Ortuno A, Nieto-Munera J, Lopez-Pina JA. Effectiveness of an educational physiotherapy and therapeutic exercise program in adult patients with hemophilia: a randomized controlled trial. Arch Phys Med Rehabil. 2017; 98:841-848.

17. Schmid A, Duncah PW, Studenski S, Lai SM, Richards L, Perera S, Wu SS. Improvements in speed-based gait classification are meaningful. Stroke. 2007; 38:20962100.

18. Greene WB, Strickler EM. A modified isokinetic strengthening program for patients with severe hemophilia. Dev Med Child Neurol. 1983; 25:189-196.

Received March 8, 2021; Revised June 2, 2021; Accepted July 15, 2021.

Released online in J-STAGE as advance publication July 29, 2021.

\section{*Address correspondence to:}

Junko Fujitani, Department of Rehabilitation Medicine, National Center for Global Health and Medicine, 1-21-1 Toyama Shinjuju-ku, Tokyo 162-8655, Japan.

E-mail: jufujita@hosp.ncgm.go.jp 